\section{Simulation and In Vitro Experimental Studies on Targeted Photothermal Therapy of Cancer using Folate-PEG- Gold Nanorods}

\author{
Shayan Maleki ${ }^{1 \oplus}$, Mohammad Farhadi², Seyed Kamran \\ Kamrava², Alimohamad Asghari ${ }^{3}$,Ahmad Daneshi ${ }^{*} \odot$
}

\begin{abstract}
Background: Selective targeting of malignant cells is the ultimate goal of anticancer studies around the world. There are some modalities for cancer therapy devastating tumor size and growth rate, meanwhile attacking normal cells. Utilizing appropriate ligands, like folate, allow the delivery of therapeutic molecules to cancer cells selectively. There are a variety of photosensitizers, like gold nanorods (GNRs), capable of absorbing the energy of light and converting it to heat, evidently build a photothermal procedure for cancer therapy.
\end{abstract}

Objective: To develop a one-step approach for calculating the temperature distribution by solving the heat transfer equation with multiple heat sources originating from NIR laser-exposed GNRs.

Material and Methods: In this experimental study, we simulated NIR laser heating process in a single cancer cell, with and without incubation with folate conjugated PEG-GNRs. This simulation was based on a real TEM image from an experiment with the same setup. An in vitro experiment based on aforesaid scenario was performed to validate the simulated model in practice.

Results: According to the simplifications due to computational resource limits, the resulting outcome of simulation showed significant compatibility to the supporting experiment. Both simulation and experimental studies showed a similar trend for heating and cooling of the cells incubated with GNRs and irradiated by NIR laser ( 5 $\min , 1.8 \mathrm{~W} / \mathrm{cm}^{2}$ ). It was observed that temperature of the cells in microplate reached $53.6{ }^{\circ} \mathrm{C}$ when irradiated by laser.

Conclusion: This new method can be of great application in developing a planning technique for treating tumors utilizing GNP-mediated thermal therapy.

Citation: Maleki Sh, Farhadi M, Kamrava SK, Asghari A, Daneshi A. Simulation and In Vitro Experimental Studies on Targeted Photothermal Citation: Maleki Sh, Farhadi M, Kamrava SK, Asghari A, Daneshi A. Simulation and In Vitro Experimental Studies on Targ
Therapy of Cancer using Folate-PEG-Gold Nanorods. J Biomed Phys Eng. 2021;11(4):435-446. doi: 10.31661/jbpe.v0i0.1108.

\section{Keywords}

Computer Simulations; Hyperthermia, Induced; Theranostic Nanomedicine; GNR-PEG-Folate; Dose Enhance; Cancer; Heat Transfer

\section{Introduction}

he main goal of employing nanoparticles as the operative agent is to induce hyperthermia. The ability of nanoparticles to infiltrate biological cells without being captivated by immune system is the gold advantage of their ultra-small size. On the other hand, the general consequence of utilizing nanoparticles is the long-term
${ }^{1} \mathrm{PhD}, \mathrm{ENT}$ and Head \& Neck Research Center and Department, Hazrat Rasoul Hospital, the Five

Senses Institute, Iran

University of Medical Sci-

ences, Tehran, Iran

${ }^{2} \mathrm{MD}$, ENT and Head \&

Neck Research Center

and Department, Hazrat

Rasoul Hospital, the Five

Senses Institute, Iran

University of Medical Sci

ences, Tehran, Iran

${ }^{3} \mathrm{MD}$, Skull Base Re-

search Center, the Five

Senses Health Institute,

Iran University of Medi-

cal Sciences, Tehran,

Iran

Corresponding author: Ahmad Daneshi

ENT and Head \& Neck

Research Center and

Department, Hazrat

Rasoul Hospital, the Five Senses Institute, Iran

University of Medical Sci-

ences, Tehran, Iran

E-mail: daneshiahmad@ gmail.com

Received: 10 January 2019 Accepted: 28 February 2019 
safety for human organs, which has attracted huge attention from specialists. As this part of nanotechnology application is still newfound, there are not enough convincing evidences to ensure health-care authorities around the world for any mid- or long-term effects of these newborn particles on human organs. To minimize any unwanted effects, specialist's recommendation would clearly be "does enhancement" [1-3]. Dose enhancement of local or systematic application of nanoparticles has attracted a great deal of attention as expected. Invention of magnetic absorption of metallic nanoparticles to tumor site is an example of augmented endeavor towards dose enhancement. Selective targeting of tumor cells to motivated tumor uptake of active nano-agents by advanced nanoparticle bundles is another effort of scientists to minimize the dose and amplify therapeutic effects substantially.

Other modalities have been active in cancer confrontation around the world for years. Particle beam therapy, including alpha therapy, charged particle therapy as ions, protons, and neutrons, to chemotherapy and surgical resection and tissue drainage have clear advantages and their exclusive disadvantages. Based on evidences, some of the incident energy in these approaches is released in healthy tissue, resulting in ionization of molecules, DNA damage and development of secondary tumors in some cases. Although many efforts have been concentrated on innovating new technologies to minimize side effects by controlling radiation dose or developing new procedures, less prosperity is achieved and those successful approaches are too much expensive. On the other hand, recent synthetic chemical drugs have shown promising improvement in an overall decrease of detrimental side effects. Avastin ${ }^{\circledR}$, Rituxan ${ }^{\circledR}$, Herceptin ${ }^{\circledR}$, Xeloda $^{\circledR}$, Tarceva $^{\circledR}$ (Roche), Revlimid ${ }^{\circledR}$ (Celgene), Imbruvica ${ }^{\circledR}$, Velcade $^{\circledR}$, Zytiga $^{\circledR}$ (Johnson \& Johnson), Gleevec ${ }^{\circledR}$, Afinitor ${ }^{\circledR}$, Tasigna ${ }^{\circledR}$ (Novartis) and Erbitux ${ }^{\circledR}$, Yervoy ${ }^{\circledR}$ (BMS) are among the top global demands of chemos in recent years. Recently, it is found a huge drop in chemotherapy prescriptions for some early stage cancers in stages 1 to 2 . A recent study revealed that from 2013 to 2015, oncologists prescribe chemotherapy for a fifth of early stage of breast cancer candidates, which was over a third of candidates back from 2013. The oncologists have revealed implications on the overall perception and awareness of overtreatment of chemos, whilst acceptance and implication of recurrence predictors like MammaPrint ${ }^{\circledR}$ and OncotypeDx ${ }^{\circledR}$ are outbreaking [4]. Oncologists are increasingly prescribing more advanced drugs, targeting tumors with specific molecular aberrations that many of them are prepared in form of pill. There are many examples, including hormone-blocking agents, targeting cancers of breast and prostate, inhibitors of proteins from change or amplification, such as EGFR or ALK in lung cancer, and PARP drugs in cancers of ovarian in addition to some forms of breast cancer recently approved. Reports are reflecting a trend of decreasing chemotherapy use in comparison with antibody, resulting in a revolutionary conversion in treatment of cancer and other types of malignancy.

In clinical practice, the term "Hyperthermia" is used when the temperature of the target tissue increases between 40 and $45^{\circ} \mathrm{C}$ by any means (mild hyperthermia $40-42{ }^{\circ} \mathrm{C}$ and high hyperthermia $>50^{\circ} \mathrm{C}$ ). This temperature can damage and kill cells if hold for appropriate period. The correlation of temperature and time resulting in hyperthermia is calculated by Thermal Dose equation [5-8]. The key advantage in utilization of Hyperthermia is heating targeted tissue without damaging normal cells. Hyperthermia has produced significant advantages in clinical procedures especially on eliminating tumor tissue [9-11]. Several clinical trials have demonstrated the combination of hyperthermia with other interventions as radiotherapy. To achieve hyperthermia, several techniques such as infrared illumination, focused ultrasound, microwave heating, 
induction heating, and magnetic induction are utilized. Nano-particle assisted hyperthermia has achieved a good attention among scientists, especially when was stimulated with near-infrared (NIR) light.

Novel agents which can bring heat source to subcellular level and induce precise selective hyperthermia, are nano-particles in form of synthesized nano-bundles. Nano-particles are characterized with their plasmonic resonance frequency. At the production level, nano-particles can be designed to absorb electromagnetic energy in a specific wavelength. In medical applications, this frequency is generally set in near infrared region where tissue absorption is minimum.

As a brief explanation, the incident electromagnetic wave, as sensed by conduction electrons of metallic nano-particles, causes a displacement and the attraction nucleus Coulomb force produces an oscillation. This displacement is referred to as Polarization in atomic physics. Density of electron cloud, physical parameters of electron cloud around nucleus, and the effective electron mass can change the frequency of this oscillation [12]. Considering nano-particles with scales much smaller than the wavelength of the incident electromagnetic wave, the atomic polarization characterized by the dipole mode of oscillation is the dominant factor prescribing SPR frequency. Shape and size of nano-particle affect the dipole mode, more than the type of metal [13]. These parameters can be set in nano-particle synthesis processes; thus, we would be able to tune SPR frequency to a wide range of frequencies in laboratory. Considering the shape, isotropic NPs reveal one SPR frequency, and anisotropic NPs have more than one characteristic frequency.

The SPR frequency possesses an intrinsic absorption process for nano-particles, converting the absorbed electromagnetic energy to heat via a non-radiative relaxations process on the order of picoseconds. This heating mechanism is widely used in today's medical research. By selectively accumulating and targeting malignancies [14], nano-particle induced hyperthermia has overcome the failures of other modalities.

\section{Material and Methods}

This experimental study is characterized in two main sections: experiment and simulation. Primarily we discuss the experiment approach. Afterwards the modeling portion would be presented, followed by an analogy between these two parts.

\section{Nanoparticle Preparation \\ Materials}

PolyEthyleneGlycol-amino (SH-PEG-NH ${ }_{2}$, Mw 1,000) thiol was provided from Creative PEGWorks company (Winston Salem, NC, USA).

$\mathrm{KB}$ cancer cells was provided from the Iranian Pasteur institute (Tehran Branch).

\section{Synthesis (GNR)}

\section{Seed Preparation}

An aqueous solution $(20 \mathrm{~mL})$ comprising of $\mathrm{HAuCl}_{4}\left(2.5 \times 10^{-4} \mathrm{M}\right)$ and tri-sodium citrate $\left(2.5 \times 10^{-4} \mathrm{M}\right)$ was diluted in a taper flask. An ice-cold solution of $\mathrm{NaBH}_{4}$ solution $(0.6 \mathrm{~mL}$, $0.1 \mathrm{M}$ ) was suddenly added to the solution while being stirred. After $\mathrm{NaBH}_{4}$ was added to the solution, it turned pink immediately. The color change indicates the formation of nanoparticles in the solution. After 2 to 5 hours passed preparation, the particles would be used as seeds in the following steps [15] (supplementary information of the article).

\section{Preparation Nano-Rod}

Growth solution $(10 \mathrm{~mL})$ prepared from $\mathrm{HAuCl}_{4}\left(2.5 \times 10^{-4} \mathrm{M}\right)$ and cetyltrimethylammonium bromide (CTAB) $(0.1 \mathrm{M})$ was added to a solution of ascorbic acid $(0.05 \mathrm{~mL})$ and stirred thoroughly in a clean flask. Then the prepared seed solution was added $(0.025$ $\mathrm{mL}$ ) and agitation and stirring was stopped. A change in the color of the solution to reddish brown was detected after 10 minutes [16] (supplementary information of the article). 
III. Functionalized Gold NanoRods

We sequentially mixed FA (10.5 mg), EDC. $\mathrm{HCl}(9.2 \mathrm{mg})$, and NHS (5.5 mg) and added the mixture to PBS $\left(20 \times 10^{-3} \mathrm{M}, 20 \mathrm{~mL}, \mathrm{pH}\right.$ 7.4). The solution was stirred for $4 \mathrm{~h}$ in a dark place and at room temperature. This solution was added to an aqueous solution of SH-PEG$\mathrm{NH}_{2}$ (Mw 1000) and stirred for $24 \mathrm{~h}$ at room temperature and in dark. To extract the extra free FA, NHS, and EDC, we used a $3500 \mathrm{Da}$ dialysis and purified the solution using PBS $\left(20 \times 10^{-3} \mathrm{M}, \mathrm{pH} 7.4\right)$ for 3 days. The purified solution was dried to result in SH-PEG1000FA [15] (supplementary information of the article).

To achieve the final product, we added the resulting SH-PEG1000-FA to the solution of GNRs $\left(20 \times 10^{-3} \mathrm{M}, 20 \mathrm{~mL}, \mathrm{pH} 8.0\right)$. The solution was stirred in the dark and at room temperature for $24 \mathrm{~h}$ and suddenly centrifuged $\left(1.2 \times 10^{4} \mathrm{rpm}\right)$ for $30 \mathrm{~min}$. The resulting product was washed with ultrapure water for three times and then was dried under vacuum pressure to obtain the final solid product.

\section{Nanoparticle Characterization \\ TEM images}

To characterize the product, we used a ZEISS EM900 transmission microscope. After taking the extra water out of sample and drying it, the following images was yielded (Figure 1).

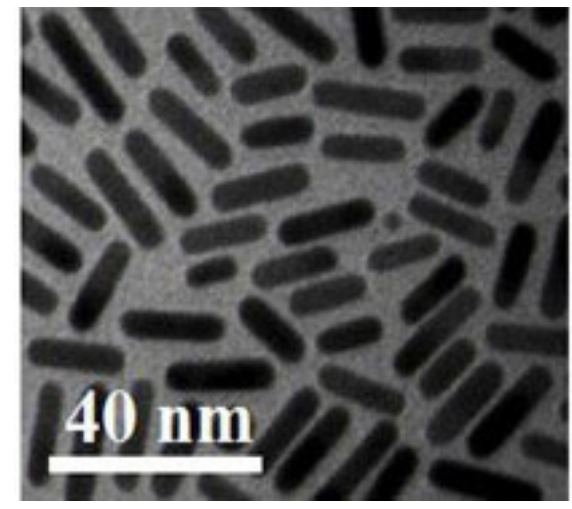

Figure 1: Transmission electron microscopy (TEM) image of folic acid conjugated gold nanorod (GNR-FA)
UV-Visible Absorption Spectroscopy

To examine the product with a UV-Visible Spectrophotometer, we filled the machine with 500 ul of nano-product and stowed the rest with distilled water.

Here, we can calculate the size of our particles based on Gans' theory (an extension of Mie's theory), which in its simplified form, estimates the NanoRod dimension using the longitudinal surface Plasmon peak as:

$R=\frac{\text { Longitudinal Absorption Peak } \lambda(\mathrm{nm})-381.49}{97.56}(1)$

$\mathrm{R}$ is the ratio of length to width of GNR in 2D TEM images. As shown in Figure 2, the longitudinal absorption peak is placed on $\sim 810 \mathrm{~nm}$ :

$$
R=\frac{810-381.49}{97.56}=4.39
$$

According to TEM images, this estimated ratio is actually acceptable.

\section{Experiment}

$\mathrm{KB}$ cancer cells proliferated and passaged up to 100.000 cell counts to feed the experiment. Cytotoxicity of GNR-PEG-FA was investigated using nanoparticles of two concentrations: 10 and $20 \mu \mathrm{g} / \mathrm{ml}$. The solutions were incubated for four hours against control group of $\mathrm{KB}$ cells without nanoparticles. Eight wells were dedicated to each group containing 5,000 cells each. Cell survival was determined using MTT assay.

After 4 hours of incubation with $10 \mu \mathrm{g} / \mathrm{ml}$ of

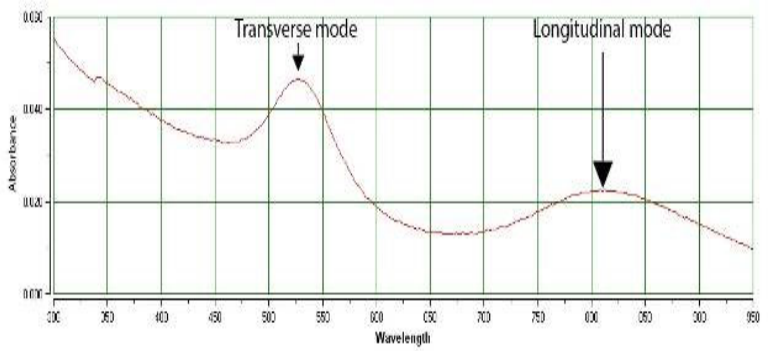

Figure 2: Ultraviolet (UV)-Visible spectroscopy of folic acid conjugated gold nanorod (GNR-FA) 
nanoparticles, the residuals were washed out with PBS then trypsinized, centrifuged and re-suspended with $100 \mathrm{ml}$ of PBS. The solution was then transferred to Eppendorf tubes and aqua-regia ( $\left.\mathrm{HCl}: \mathrm{HNO}_{3}=3: 1\right)$ was added to perform a complete digest of cells. To measure the gold contents in pictogram of AU/ cell, ICP-MS was performed by a spectrometer (ELAN DRC-e) as described (PerkinElmer SCIEX $^{\mathrm{TM}}$, Concord, ON, Canada) [12].

To examine the thermal effects of laser activated GNR-PEG-FA, eight wells of 12,000 cell counts each were incubated with nanoparticles for 4 hours, as cases and other eight wells free of nanoparticles as controls. The laser was chosen to be an $808 \mathrm{~nm}$ wavelength with a continuous output power of 1.8 Watts $/ \mathrm{cm}^{2}$. The laser system was produced and characterized by Nanobon company, Iran. The wells were illuminated for 5 minutes and temperature changes were investigated using a Testo 875i IR-Camera, Germany. Temperature changes were recorded every 60 seconds and this data acquisition continued for 5 minutes after laser radiation was turned off.

\section{Modeling}

The aim of modeling and simulation of nanoparticle heating impact on cellular level is to develop a reasonable and still simple paradigm to estimate and enhance the dose of nanoparticles injected in biological studies. To improve the accuracy of this estimate, we get benefit of TEM images of our experiment, and also augment it with various other homogenous studies. Comsol ${ }^{\circledR}$ Multiphysics and its heat transfer module is used as the modeling software. The shape of medium (KB cell) and GNR distribution are mimicked from TEM images to simulate real conditions more accurately. The physical properties of the medium (cell) was estimated to be that of water because it is the dominant substance in the cell medium by two-thirds, although other substances like proteins, lipids and carbohydrates are essential building blocks of a cell.
The overall pathway in the modeling of this in vitro problem contains three levels. 1) Cell level, 2) single well of a cell-culture-plate 3) real $8 \times 6$ cell-culture-plate. This model can simulate the real laboratory strategies more accurately.

\section{Cell Level}

Cell and its contaminant GNRs were rebuilt from TEM images (Figure 3-left) into a 2D vector drawing (Figure 3-right) and imported to $\mathrm{Comsol}^{\circledR}$ Multiphysics. The 2D drawing would also be used to build the 3D geometry of cell and its GNR contaminants, assuming GNRs as 3D cylinders and the cell as a $3 \mathrm{D}$ buildup of 2D TEM images by adding an axis of symmetry and some imaginations. We would present the 3D simulation of laser heating of cells containing GNR in the next study. Based on the Lambert-Beer law, Dirichlet boundary, and time dependent heat transfer equation, we were to model inter-cellular laser heating and deheating in the presence of GNRs; thus, we have taken advantage of heat transfer, EWBE, and PDE modules in Comsol ${ }^{\circledR}$ Multiphysics.

To work out this model, it is primarily divided into two concatenated sections. In the first section, the problem is solved for energy

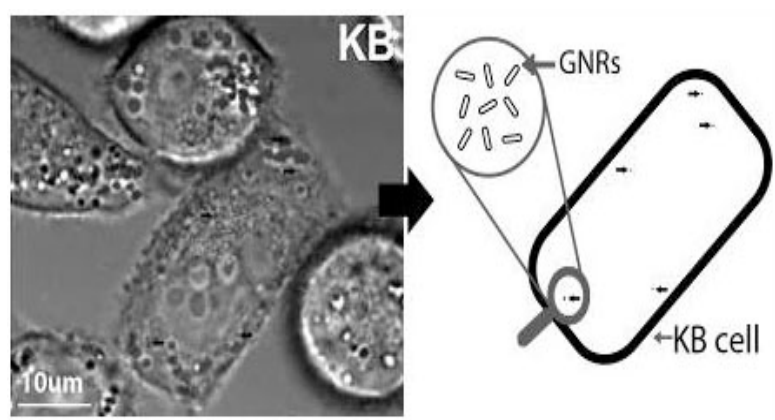

Figure 3: (left) Transmission electron microscopy (TEM) image of KB cell in the presence of Gold Nanorod (GNR). (right) A model of KERATIN-forming tumor cell line HeLa (KB cell) in the presence of GNR, drawn based on TEM image (left). (black arrows show the GNRs position in a sample KB cell). 
absorbed in the cell medium; the second is solved for energy absorbed in GNRs.

To solve the first section, we considered the laser light as a single wavelength, collimated wave $(808 \mathrm{~nm}$ laser source, which was the available source in our laboratory), experiencing minimal reflection, refraction and scattering within the medium. This way LambertBeer law can be applied to absorbed energy within medium as described in equation 3 .

$$
\frac{\partial I}{\partial z}=a(T) I
$$

Where I represent the laser intensity, $\mathrm{z}$ the laser direction traversing in the medium, and $\mathrm{a}(\mathrm{T})$ the temperature-dependent coefficient of absorption. As this temperature can vary in time and location, temperature distribution of the illuminated medium should also be solved (4).

$$
\rho c_{p} \frac{\partial T}{\partial t}+\rho c_{p} u . \nabla T=k \nabla^{2} T+Q(t)
$$

$\mathrm{Q}(\mathrm{t})$ is the heat source $\left(\mathrm{cal} / \mathrm{s} . \mathrm{cm}^{2}\right)$ coming from absorbed laser energy by the medium and Nanoparticles; $\rho$ and $c_{p}$ are the tissue density $\left(\mathrm{g} / \mathrm{cm}^{3}\right)$, specific heat $(\mathrm{kcal} / \mathrm{kg} . \mathrm{K})$, respectively. $\mathrm{u}$ and $\mathrm{k}$ are the medium turbulent velocity $\left(\mathrm{m} / \mathrm{s}^{2}\right)$, thermal conductivity $(\mathrm{kcal} /(\mathrm{h} \mathrm{m}$ $\mathrm{K})$ ), and $\mathrm{t}$ represents time(s).

To implement Lambert-Beer law in $\mathrm{Comsol}^{\circledR}$ Multiphysics, we make the use of a general form partial differential equation (PDE) and set the source term as equation 5 by considering a linear relation between a $(\mathrm{T})$ and $\mathrm{T}$ :

$$
a(T)=a_{0}+c T ; c=\frac{\partial a(T)}{\partial T}
$$

According to a study on temperature dependent absorption of light in various wavelengths in water, carried out by Langford et al., we can consider $\mathrm{x}$ to be $-133 \times 10^{-5}\left(\mathrm{~m}^{-1} \mathrm{~K}^{-1}\right)$ and estimate $a_{0}$ to be 1.95 around wavelength 808 $\mathrm{nm}$. The temperature in our project is $30-60$ ${ }^{\circ} \mathrm{C}$. By considering mean value of $a_{0}$ at this range, the source term in PDE can be written as equation 6 by solving for $\mathrm{f}=0$ :

$$
f=\frac{\partial I}{\partial r}-[1.95-133 T] \times I=0
$$

As the laser light experiences many scatterings in the way reaching our cell surface, we consider a uniform laser energy distribution at the surface and Dirichlet boundary condition would impose a constant number. We consider the laser output energy stable and thus we would consider input heat flux in Newmann boundary condition to be zero when solving the heat distribution part.

To calculate time dependent temperature variations, water thermal properties, summarized in Table $1[6,7]$, are used as input to equation 4 . The heat source $\mathrm{Q}(\mathrm{t})$ results from laser intensity times absorption coefficient at each location, assuming that the generated thermal energy (heat) in a small thickness ' $z$ ' represents the absorbed laser energy as in equation 3.

For the second section, we consider GNRs to generate heat by SPR in a homogenous medium, and the temperature variations at location $\vec{r}$ relative to GNR at location $\vec{r}_{n}$ is described by the equation of heat conduction [17]:

$$
\Delta T(\vec{r})=\frac{\sigma \times \varphi\left(\vec{r}_{n}\right)}{4 \pi k\left|\vec{r}-\vec{r}_{n}\right|}
$$

Where $\sigma$ is GNR absorption cross section, $\varphi\left(\vec{r}_{n}\right)$ is the laser power at GNR location (W/ $\mathrm{m}^{2}$ ), and $k$ is the thermal conductivity of the surrounding medium (W/K.m). To solve this part, we used a technique described by Cheong et al, [18]. Also, Discrete dipole approxima-

Table 1: Water thermal properties.

\begin{tabular}{ccc} 
& Value & Unit \\
\hline Density & 0.322 & $\mathrm{~g} / \mathrm{cm}^{3}$ \\
\hline Specific heat & 0.5035 & $\mathrm{kcal} / \mathrm{kg} . \mathrm{K}$ \\
\hline Turbulent velocity & 0 & $\mathrm{~m} / \mathrm{s}^{2}$ \\
\hline $\begin{array}{c}\text { Thermal conductivity } \\
\text { (at } 40^{\circ} \mathbf{C} \text { ) }\end{array}$ & 0.5405 & $\mathrm{kcal} /(\mathrm{h} \mathrm{m} \mathrm{K})$
\end{tabular}


tion (DDA) method [19] was used to estimate $\sigma$ for GNRs. As generally described for undefined-shape particles, effective radius can be estimated by:

$$
r_{e f f}=(3 V / 4 \pi)^{1 / 3}
$$

Where $V$ is the particle volume. Effective radius in equation 8 represents the radius of a virtual sphere with a volume equal to that of our particle. For nanorod particles, aspect ratio $(\mathrm{R})$ is another factor defining absorption cross section, previously referred in equation 2 . Jain et al., [20] have measured $\mathrm{R}$ to be $\sim 4.6$, and $\mathrm{r}_{\text {eff }}$ $11.43 \mathrm{~nm}$ for one of their GNRs similar to our nanoparticles. Considering Jain et al. experiment, the value for $\sigma$ can be calculated to be $6,255 \mathrm{~nm}^{3}$.

Laser power at GNR location, $\varphi\left(\vec{r}_{n}\right)$, is worked out by utilizing light-diffusion-approximation equation described by Nyborg et al, [12]. Considering a continuous laser source, $\varphi\left(\vec{r}_{n}\right)$ is written as:

$$
\varphi\left(\vec{r}_{n}\right)=\frac{P_{0} \exp \left(-\mu_{e f f} \vec{r} \cdot \hat{n}\right)}{4 \pi D r}
$$

Where $P_{0}$ is the incident light power, $\mu_{\text {eff }}=\sqrt{3 \mu_{\mathrm{a}}\left(\mu_{\mathrm{a}}+\mu_{s}^{\prime}\right)}$, is the effective coefficient of attenuation, and is the beam direction, $\mu_{\mathrm{a}}$ is the coefficient of absorption, $\mu_{s}^{\prime}$ is the reduced coefficient of scattering, and $D=1 / 3\left(\mu_{\mathrm{a}}+\mu_{s}^{\prime}\right)$ is the diffusion coefficient of the medium.

Concatenation of section one and two, results in an accurate temperature calculation for the cell environment, containing a number of GNR particles and bearing laser energy illumination. Therefore, we would be ready to step forward to the next level.

\section{Single Well}

Modeling this approach in a single cell has enabled us to simulate temperature variations for a well of ordinary cell-culture-plate containing various amounts of cells. Following our experiment, each well of cell-culture-plate contains 12,000 of KB cells. To model this amount, we considered a homogeneous distribution of cells in a circular well of a typical cell-culture-plate $0.9 \mathrm{~cm}$ in diameter. Then an assembly of 12,000 cells distributed in a symmetric quadrant was calculated, considering two axes of symmetry as displayed in Figure 4a. In this approach, each cell and its contaminant GNRs were considered as a single object with characteristics primarily calculated in single cell level. This technique, in addition to circular symmetric of model model, have reduce computational time, load, and costs. The overall model is assembled by joining 4 of these quadrants in a proper way presented in Figure 4b.

\section{Cell-Culture-plate}

By calculating for a single well of a cell-culture-plate containing $12,000 \mathrm{~KB}+\mathrm{GNRs}$, it is relevant to simulate a typical $8 \times 6$ cell-cultureplate. Forty-eight single wells are arranged in an $8 \times 6$ matrix (Figure $4 \mathrm{c}$ ). The model is solved in $\mathrm{Comsol}^{\circledR}$ multiphysics for polystyrene as the plate material. To be analogous to experimental setup, only a single well among forty-eight received laser illumination, and

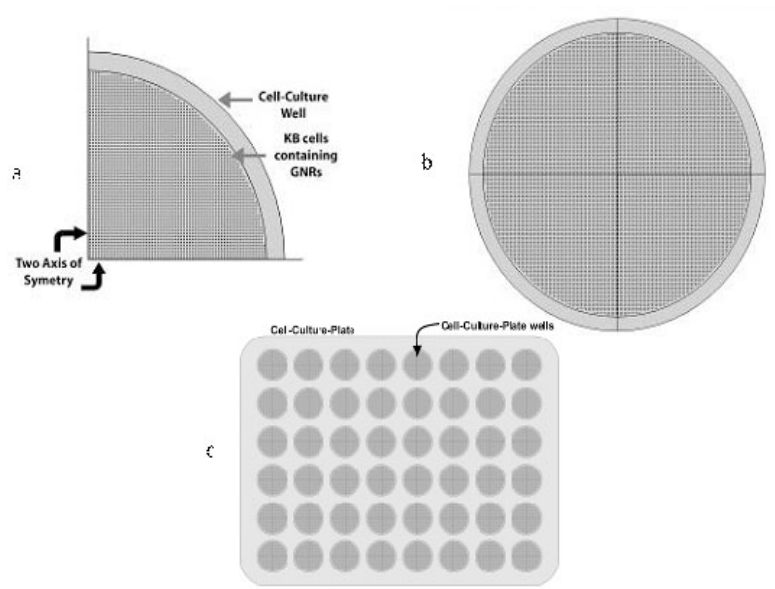

Figure 4: (a) symmetric quadrant containing 3,000 KB+gold nanorods (GNRs), (b) 4 quadrants joint to build a cell-culture-plate well, (c) cell-culture-plate model. 
other wells and the overall cell-culture-plate was considered to perform realistic boundary conditions. In this simulation, as in compliance to experiment, the incident light power $\left(P_{0}\right)$ was considered to be 1.8 Watts $/ \mathrm{cm}^{2}$ for the first 5 minutes and 0 for the next 5 minutes.

\section{Results}

Modeling

Based on ICP-MS and TEM images of GNR uptake and distribution in KB cells, a model was generated (Figure 3-right) and fed to Comsol ${ }^{\circledR}$ multiphysics for heat generation and transfer as described previously. The simulation is divided into three levels as described earlier: 1) Cell level, 2) single well of a cellculture-plate 3) Cell-culture-plate $8 \times 6$.

The simulation was run based on previously mentioned setup for five minutes in the presence of laser heating and other five minutes considering the laser heating are turned off. The simulation outcome is described here for each level.

1) Cell level: Cell containing GNRs

To simulate the impact of GNRs on temperature fluctuations, heat distribution analysis is performed. For demonstrate purposes, a separate calculation is also done in microsecond time scale (Figure 5a).

Figure 3-right shows the cell-GNRs setup imitating TEM image (Figure 3-left) due to the use of it in our simulation. Five bundles of GNRs were placed in TEM proposed positions in a semi-cubic environment (KB cell) bearing a laser power irradiation of 1.8 Watts $/ \mathrm{cm}^{2}$. This radiation also heats the $\mathrm{KB}$ cell and surrounding environments. The heat generation and distribution formulas for GNRs and KB cell were implemented to Comsol ${ }^{\circledR}$ Multiphysics, including two time dependent study steps, one for laser-on for a period of 5 minutes, and the other for laser-off for another period of five minutes.

As seen in Figure 5a, the deployment of nanoparticle clusters can amplify laser energy concentration in the near neighborhood medium. Moreover, as GNRs infiltrate onto the membrane of a cell, they can precisely act as an ultimate target to destruct cell membrane. As we extend time scale and study this model in minutes, heat conduction and distribution lead into the medium to an equilibrium situation. A time dependent profile is demonstrated in Figure 5 in minute scale.

Laser is turned on at time zero and turned off at fifth minutes. Temperature change is followed-up for five minutes after laser power off.

2) Single well of a cell-cultureplate

At the next level, the simulation was set to calculate the accumulation of temperature changes in 12,000 cells. Each cell was put to neighbor 8 other cells in a flat domain of 2 dimension (Figure 5c). The time steps between images are 60 seconds each. Maximum and average temperature are plotted for every time step in Figure 5b.

As seen in Figure 5b, the cell-GNRs are distributed in cell-culture well homogeneously. Each field is divided into three regions. Inner circle with a radius of $0.9 \mathrm{~cm}$ diameter and outer circle $1.0 \mathrm{~cm}$ diameter (Figure 5c). Furthermore, as laser is turned off, temperature
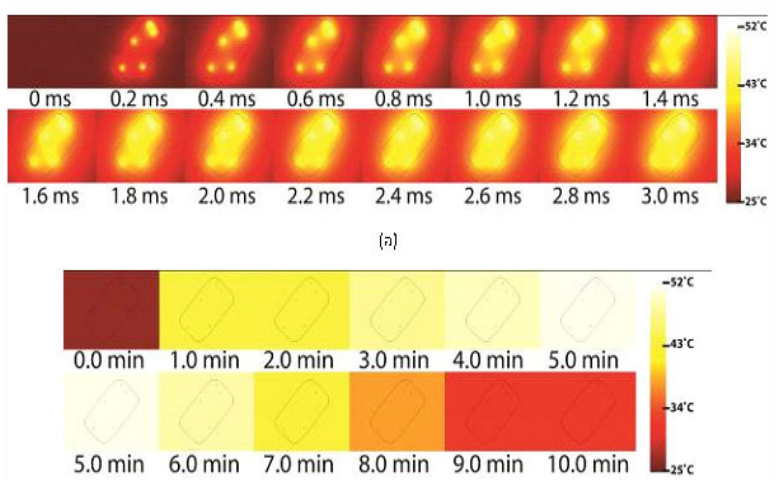

(b)

Figure 5: (a) Demonstration of micro-second period temperature variations, (b) demonstration of minute period temperature variations 
Hyperthermia Induced by GNR Mediated Photothermal Therapy

drops rapidly and more rapidly as getting distant from center.

3) Cell-culture-plate

Simulating a real $8 \times 6$ cell-culture-plate was programmed in this level. As only one well of the plate is illuminated, we illustrate the nearest neighbors of illuminated well, resulting in a $3 \times 3$ matrix of wells of a cell-culture-plate (Figure 6a). In Figure 6a and b, upper row is for laser-on period, and lower ones are modeled when the laser power was turned off. The heat deposition in cell-culture-plate is playing a great role in temperature decrease over illuminated well.

To evaluate GNRs impact on heat generation and temperature rise, we have calculated the same assembly in absence of GNRs. The previously mentioned triple-level construction is also repeated in absence of GNRs but is not included here and only the third level is demonstrated in Figure 6b.

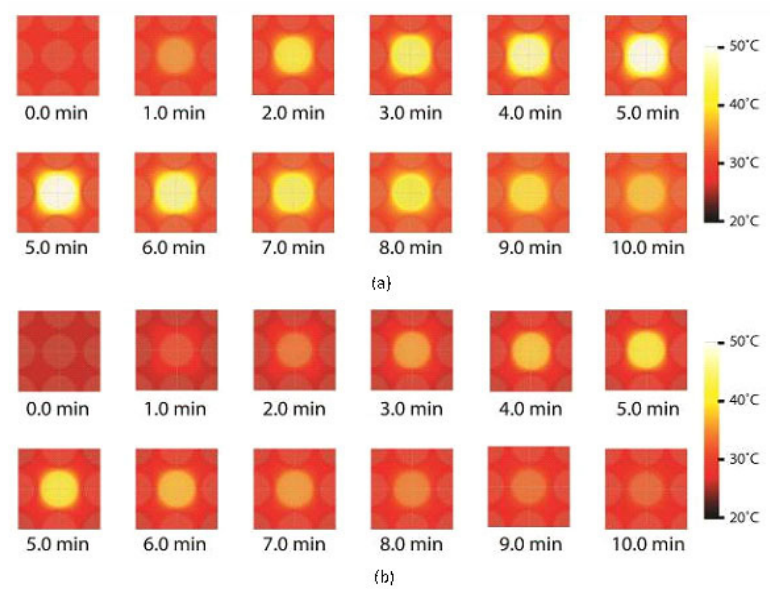

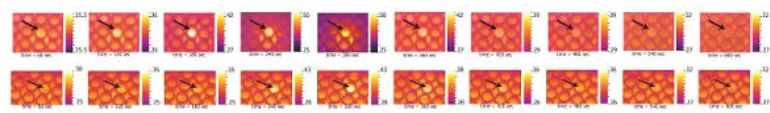

(c)

Figure 6: Demonstration of minute period temperature variations in cell-cultureplate level (a) in presence of gold nanorods (GNRs), (b) in absence of GNRs in simulation, and (c) in presence (up) and absence (down) of GNRs in experiment.

\section{Experiment}

All wells of an $8 \times 6$ cell-culture-plate was filled with $12000 \mathrm{~KB}$ cells. Half of the wells were incubated with GNRs as described before. Laser at $808 \mathrm{~nm}, 1.8 \mathrm{Watt} / \mathrm{cm}^{2}$ was directed on a single well for 5 minutes and temperature changes were recorded by a Testo ${ }^{\circledR}$ thermal-camera. After 5 minutes, laser was turned off and temperature was followed up for an extra 5 minutes to retrieve cooling conditions.

Figure $6 \mathrm{c}$ shows a time-lapse of temperature for laser radiated $\left(808 \mathrm{~nm}, 1.8 \mathrm{~W} / \mathrm{cm}^{2}\right) \mathrm{KB}$ cells with and without GNRs. The images are taken with a Testo ${ }^{\circledR}$ thermal camera. In addition, the highest temperature of each lapse is extracted and plotted in Figure 7.

\section{Discussion}

In the domain of thermal therapy, the action is performed mainly by heating directly or the use of an intermediate agent, and the ability to predict temperature changes would be highly important. Heating the target over or under a targeted operational temperature (thermal dose) would result in undesirable effects. Under-heating would not allow a desired treatment to occur, as the temperature is not

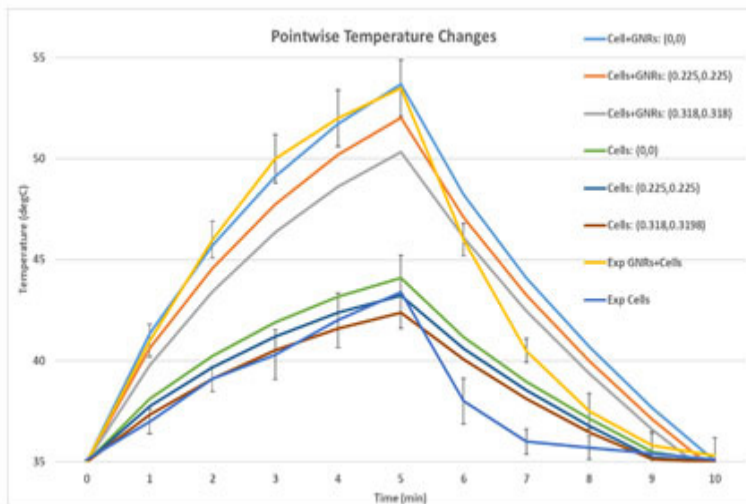

Figure 7: Temperature curve of three points with different distances from center of the illuminated well in our simulated cell-cultureplate. (green: center; blue: $r=0.3 \mathrm{~cm}$; red: $r=0.45 \mathrm{~cm}$ ) for Cells+gold nanorods (GNRs) and Cells separately compared to experiment (Exp). 
enough for a certain thermal dose given in the treatment period. On the other hand, overheating, results in adverse effects on neighboring healthy cells $[13,21]$. Modeling and simulation have a great contribution among prediction methods, although living systems are so complicated and require many resources to reach an enough reliable result. To simulate living systems precisely, an accurate yet fluent model should be employed to prohibit missing of minute yet important details. In spite of some discrepancies with our experiment, we setup a two-dimensional computational model covering both microscopic and macroscopic fields. The model has three levels and an inductive (from bottom to top) structure, bringing into account nano-rods position in cells as well as cell-culture-plate material properties. These properties play a great role in mathematical calculations of optical and thermal modeling. In the case of gold-nano-rods, it is well-known that elongating nanoparticles to rod shape, affecting physical characteristics widely. An advantage of rod shape gold nanoparticles (GNRs) is in their second absorption band, compared to gold-nano-spheres. GNRs, based on their double axis nature, possess a major axis, which accordingly absorbs longer wavelengths in infra-red spectrum which is due to the plasmonic resonance on their major axis [22-24]. This second band is attractive to biomedical application, where a common request to light penetration improvement is highlighted.

To result in a correct modeling of this project, we divided the assembly into three levels, which is arranged from nano-scale up to macro-scale design. To be identical to real conditions, we estimated the distribution of GNRs in our simulated KB cells by the help of TEM images taken from our experimental setup (Figure 3-left). In this level of analysis, the analytical simulation was divided into two parts: GNRs and a single cell. The GNRs calculations were performed based on the equation of heat conduction (eq. 7); while the cell part was accomplished by the equation of temperature distribution (eq. 4). Accumulation of these two parts results in a good estimation or rather say "prediction" of microscopic temperature profile of a cell containing a number of GNRs distributed in clusters of 9 GNRs in various positions. In the next level, we zoom out from a single cell to a collection of 12,000 cells in a well of cell-culture-plate. The cells are calculated as single units of heat sources, which are the result of superposition of cell and GNRs, computed previously. The thermal conduction properties of polystyrene were also affecting this calculation in in the boundary conditions, placed as a surrounding wall of a well and the rear plate. The above boundary layer was considered as air with ambient conditions. To bring our model more identical to a real situation, the last level was designed. In this level, we emulate a real cell-culture-plate in a two-dimensional space. All materials and boundary conditions are set and the wells are positioned identical to a real $8 \times 6$ cell-cultureplate. Only one of the wells is experiencing laser illumination. Temperature changes of interior sites were fetched from previous level while exterior sites were analyzed and calculated separately. In order to reduce computational load, we made the use of symmetric axis which was available in second and third levels (Figure 4a, b).

The simulation scenario presented in this paper is arranged to provide a sensitive and almost accurate estimation of the heating prediction in a sample in vitro setup of cells with and without nanoparticles undergoing laser irradiation. A great attempt was dedicated to bring any heterogeneity found within a real biological assembly into account. Although the scenario is designed in two-dimensional space, for the sake of computational time, negligible z-axis of experiment compared to area of elements makes it a good estimate of the real world. Overall modeling and design are completely following our experimental setup to make a better comparison afterwards. Fo- 
late GNR-PEG-folate conjugate is prepared in laboratory and incubated in KB cell-cultureplate for 4 hours. After performing ICP-MS and TEM, the overall outcome was used to determine the uptake of nano-complex in $\mathrm{KB}$ cells, used in designing our simulation [25]. The results reveal that in the presence of adequate nano-particles infiltrated into a cell, heat aggregation would occur and this temperature rise would demolish the cell membrane. We know that the placement of nano-particles in the proximity of cell membrane would guarantee this effect, but neighboring clusters of nano-particles would also result in a synergic effect (Figure 5a, b).

\section{Conclusion}

We have developed an analogous computer model to that of a real experiment, which also showed a good coordination in practice. The model is suggested to help predicting in-vitro temperature changes by introducing nano-particles as very small yet effective heat sources. Illumination of Gold nano-rods with an 808 $\mathrm{nm}$ diode laser, targeting the longitudinal mode of nano-rod, would not only reduce laser costs, but also increase penetration depth of laser light. The scenario followed temperature changes after laser-off as well. As experiment confirmed, GNRs and their Plasmon resonance played a major role in overall temperature increase of the medium. Both simulation and experiment were also followed after laser exposure for five minutes, and the temperature decrease was recorded. The results and measurements are also in accordance to each other, although the correlation is not as strong to that of laser-on period. This fact shows that there are still some active parameters, which are not detected and included in our model. Indeed, three-dimensional calculations of this assembly may reduce the diversity as well.

\section{Acknowledgment}

This Activity was funded by the Iran National Science Foundation (INSF) (94010132), with special thanks.

\section{Conflict of Interest}

None

\section{References}

1. Hosseini V, Mirrahimi M, Shakeri-Zadeh A, Koosha F, Ghalandari B, Maleki S, Komeili A, Kamrava SK. Multimodal cancer cell therapy using Au@ Fe203 core-shell nanoparticles in combination with photo-thermo-radiotherapy. Photodiagnosis and Photodynamic Therapy. 2018;24:129-35. doi: 10.1016/j.pdpdt.2018.08.003.

2. Mirrahimi M, Abed Z, Beik J, Shiri I, Dezfuli AS, Mahabadi VP, Kamrava SK, Ghaznavi $\mathrm{H}$, Shakeri-Zadeh A. A thermo-responsive alginate nanogel platform co-loaded with gold nanoparticles and cisplatin for combined cancer chemo-photothermal therapy. Pharmacological Research. 2019;143:178-85. doi: 10.1016/j.phrs.2019.01.005.

3. Mirrahimi M, Hosseini V, Shakeri-Zadeh A, Alamzadeh Z, Kamrava SK, Attaran N, Abed Z, Ghaznavi $\mathrm{H}$, Nami SH. Modulation of cancer cells' radiation response in the presence of folate conjugated $\mathrm{Au} @$ Fe 203 nanocomplex as a targeted radiosensitizer. Clinical and Translational Oncology. 2019;21(4):479-88. doi: 10.1007/s12094-0181947-8.

4. Kurian AW, Bondarenko I, Jagsi R, Friese CR, McLeod MC, Hawley ST, Hamilton AS, Ward KC, Hofer TP, Katz SJ. Recent trends in chemotherapy use and oncologists' treatment recommendations for early-stage breast cancer. Journal of the $\mathrm{Na}$ tional Cancer Institute. 2018;110(5):493-500. doi: 10.1093/jnci/djx239.

5. Beik J, Asadi M, Mirrahimi M, Abed Z, Farashahi A, Hashemian R, Ghaznavi H, Shakeri-Zadeh A. An image-based computational modeling approach for prediction of temperature distribution during photothermal therapy. Applied Physics $B$. 2019;125(11):213. doi: 10.1007/s00340-0197316-7.

6. Beik J, Asadi M, Khoei S, Laurent S, Abed Z, Mirrahimi $M$, Farashahi $A$, Hashemian $R$, Ghaznavi $H$, Shakeri-Zadeh A. Simulation-guided photothermal therapy using MRI-traceable iron oxidegold nanoparticle. Journal of Photochemistry and Photobiology B: Biology. 2019;199:111599. doi: 10.1016/j.jphotobiol.2019.111599.

7. Zabanran M, Asadi M, Zare-Sadeghi A, Ardakani AA, Shakeri-Zadeh A, Komeili A, Kamrava SK, Ghalandari B. The effects of gold nanoparticles characteristics and laser irradiation conditions on spatiotemporal temperature pattern of an agar 
phantom: A simulation and MR thermometry study. Optik. 2020;202:163718. doi: 10.1016/j. ijleo.2019.163718.

8. Asadi M, Beik J, Hashemian R, Laurent S, Farashahi A, Mobini M, Ghaznavi H, Shakeri-Zadeh A. MRI-based numerical modeling strategy for simulation and treatment planning of nanoparticleassisted photothermal therapy. Physica Medica. 2019;66:124-32. doi: 10.1016/j.ejmp.2019.10.002.

9. Hashemian AR,Eshghi $H$, Mansoori GA, ShakeriZadeh A, Mehdizadeh AR. Folate-Conjugated Gold Nanoparticles (Synthesis, Characterization and Design for Cancer Cells Nanotechnology-based Targeting). International Journal of Nanoscience and Nanotechnology. 2009;5(1):25-34.

10. Shakeri-Zadeh A, Eshghi H, Mansoori GA, Hashemian AR. Gold nanoparticles conjugated with folic acid using mercaptohexanol as the linker. Journal Nanotechnology Progress International. 2009;1(1):1-44.

11. Zeinizade E, Tabei M, Shakeri-Zadeh A, Ghaznavi $\mathrm{H}$, Attaran N, Komeili A, Ghalandari B, Maleki S, Kamrava SK. Selective apoptosis induction in cancer cells using folate-conjugated gold nanoparticles and controlling the laser irradiation conditions. Artificial Cells, Nanomedicine, and Biotechnology. 2018;46(1):1026-38. doi: 10.1080/21691401.2018.1443116.

12. Movahedi MM, Mehdizadeh A, Koosha F, Eslahi N, Mahabadi VP, Ghaznavi H, Shakeri-Zadeh A. Investigating the photo-thermo-radiosensitization effects of folate-conjugated gold nanorods on KB nasopharyngeal carcinoma cells. Photodiagnosis and Photodynamic Therapy. 2018;24:324-31. doi:10.1016/j.pdpdt.2018.10.016.

13. Araya T, Kasahara K, Nishikawa S, Kimura H, Sone T, Nagae H, Ikehata Y, Nagano I, Fujimura M. Antitumor effects of inductive hyperthermia using magnetic ferucarbotran nanoparticles on human lung cancer xenografts in nude mice. Onco Targets and Therapy. 2013;6:237. doi: 10.2147/0TT. S42815. PubMed PMID: 23569387. PubMed PMCID: PMC3615880.

14. Beik J, Jafariyan M, Montazerabadi A, GhadimiDaresajini A, Tarighi P, Mahmoudabadi A, Ghaznavi $\mathrm{H}$, Shakeri-Zadeh A. The benefits of folic acidmodified gold nanoparticles in CT-based molecular imaging: radiation dose reduction and image contrast enhancement. Artificial Cells, Nanomedicine, and Biotechnology. 2018;46(8):1993-2001. doi: 10.1080/21691401.2017.1408019.

15. Cheong KH, Yi DK, Lee JG, Park JM, Kim MJ, Edel JB, Ko C. Gold nanoparticles for one step DNA extraction and real-time PCR of pathogens in a sin- gle chamber. Lab on a Chip. 2008;8(5):810-3. doi: 10.1039/b717382b. PubMed PMID: 18432353.

16. Cao SW, Fang J, Shahjamali MM, Wang Z, Yin Z, Yang Y, Boey FY, Barber J, Loo SC, Xue C. In situ growth of Au nanoparticles on Fe 203 nanocrystals for catalytic applications. Cryst Eng Comm. 2012;14(21):7229-35. doi: 10.1039/C2CE25746G.

17. Carslow HS, Jaeger JC. Conduction of heat in solids. Oxford University Press; 1986.

18. Cheong SK, Krishnan S, Cho SH. Modeling of plasmonic heating from individual gold nanoshells for near-infrared laser-induced thermal therapy. Medical Physics. 2009;36(10):4664-71. doi: 10.1118/1.3215536.

19. Draine BT, Flatau PJ. Discrete-dipole approximation for scattering calculations. J Opt Soc Am A. 1994;11(4):1491-9. doi: 10.1364/JOSAA.11.001491.

20. Jain PK, Lee KS, El-Sayed IH, El-Sayed MA. Calculated absorption and scattering properties of gold nanoparticles of different size, shape, and composition: applications in biological imaging and biomedicine. The Journal of Physical Chemistry $B$. 2006;110(14):7238-48. doi: 10.1021/jp0571700.

21. Zhu X, Feng W, Chang J, Tan YW, Li J, Chen M, Sun Y, Li F. Temperature-feedback upconversion nanocomposite for accurate photothermal therapy at facile temperature. Nature Communications. 2016;7:10437. doi: 10.1038/ncomms10437.

22. Sönnichsen C, Franzl T, Wilk T, Von G, Plessen, J. Feldmann, Wilson 0, Mulvaney P. Drastic reduction of plasmon damping in gold nanorods. Phys Rev Lett. 2002;88(7):077402. doi: 10.1103/PhysRevLett.88.077402. PubMed PMID: 11863939.

23. Bohren CF, Huffman DR. Absorption and scattering of light by small particles. Weinheim, Germany: Wiley Online Library; 1998. doi: 10.1002/9783527618156.

24. Xu Z. Optical Properties of Metal Clusters By Uwe Kreibig (I. Physikalisches Inst. der RWTH Aachen, Gmermany) and Michael Vollmer (Technische Physik Brandenburg, Germany). Springer: New York. 1994. xvii+ 532 pp. \$69.00. ISBN 0-38757836-6. J Am Chem Soc. 1996;118(25):6098. doi: 10.1021/ja955378p.

25. Mehdizadeh AR, Pandesh S, Shakeri-Zadeh A, Kamrava SK, Habib-Agahi M, Farhadi M, Pishghadam M, Ahmadi A, Arami S, Fedutik Y. The effects of folate-conjugated gold nanorods in combination with plasmonic photothermal therapy on mouth epidermal carcinoma cells. Lasers in Medical Science. 2014;29(3):939-48. doi: 10.1007/s10103013-1414-2. PubMed PMID: 24013622. 